\title{
Monitoring system of thermal stress for continuous welded rails
}

\author{
X. Duan, Z. Yu, L. Zhu \& X. Xu \\ School of Mechanical, Electrical Control Engineering, \\ Beijing JiaoTong University, China
}

\begin{abstract}
Most modern railways use the continuous welded rail (CWR). A major problem is the almost total absence of expansion joints that can create buckling in hot weather and breakage in cold weather due to rail thermal stresses. A guided wave ultrasound system has been developed to detect the thermal stress in the continuous welded rail. The system operates with three transducers, one for excitation and two for reception, to detect thermal stress based on the acoustic-elasticity principle. We can find the time delay of two receiving transducers when the guided waves transmit through the rail. Then we calibrate the system to establish the relationship between time delay and thermal stress. Finally, we can find the thermal stress by calculating the time-delay of the two receiving transducers. A large scale rail testbed has been designed to simulate the thermal stress on the real track. The system is permanently installed, powered by solar panels and uses a WCDMA network to transmit the monitoring data to the remote serve station. The system has already been installed on some high-speed railway lines in China as a field test; the results indicate that the system is stable. More work needs to be done to improve the accuracy of the detection.

Keywords: continuous welded rail, guided wave, thermal stress.
\end{abstract}

\section{Introduction}

In the development of the high-speed railway, most modern railways use the continuous welded rail (CWR). The absence of expansion joints that can create buckling in hot weather and breakage in cold weather due to the rail thermal stresses creates a major problem. Buckling occurs when the high ambient temperatures generate large compression stresses in continuously welded rails 
that ultimately make the rail unstable. It is important to develop an on-line monitoring system to monitor the state of the rail to ensure the safety of the railway transportation.

In recent years, ultrasonic guided waves have been widely used in structural health monitoring (SHM). Researchers have solved many problems, for example, in applications for testing pipe, rail, plate, etc. Lots of work and tests have been done to inspect the rail by utilizing the ultrasonic guided waves. In 2003, Li and Rose [1] described a semi-analytical finite element (SAFE) method to solve the propagation problem of ultrasonic guided wave in a waveguide with an arbitrary cross section. The phase velocity and group velocity dispersion curves of the rail are calculated in the $100 \mathrm{kHz}$ frequency range. In 2004, Rose et al. [2] utilized a rail impactor device to excite guided wave in rail and used accelerometers as sensors to analysis the preferred propagation frequencies in the rail. In 2006, Bartoli et al. [3] described the frequency dispersion curves and the attenuation curves of the rail by using SAFE method. The method is able to analyze the vibration characteristics of each mode of the rail. In 2007, Chen and Wilcox [4] studied the frequency dispersion characteristics of aluminum plate, aluminum rod and rail under longitudinal tensile stress by using traditional 3D finite element method. It was found that, in a certain range, the phase velocity increases and the group velocity decreases as tensile stress increases. In 2009, Loveday [5] analyzed guided wave propagation characteristics of a one-dimensional elastic wave guide medium in longitudinal compressive stress, using a SAFE method. An expression of the sensitivity of the velocity and the wave number of the one-dimensional elastic wave guide to the axial pressure was given. In 2010, Bartoli et al. [6] used the SAFE method to obtain the frequency dispersion curves of five main vibration modes, and analyzed the variations of the horizontal bending mode, the vertical bending mode and the torsional mode in the rail at $1 \mathrm{KHz}$. In 2012, Burger [7] describes the implementation of a system using guided waves in train rails to continuously monitor the rail for breaks. In 2013, Xu et al. [8] used a SAFE method to analyze the propagating modes of $\mathrm{CHN} 60$ rail and proposed a method to excite a particular ultrasonic guided wave mode. Previous studies have predicted changes of the dispersion properties (velocity vs. frequency, wavenumber vs. frequency) of guided waves as a function of rail stress. An ultrasonic guided wave system has been developed to detect the thermal stress in the continuous welded rail (CWR). The system is based on the acoustic-elasticity principle, which is the change of group velocity is the function of the thermal stress. The biggest challenge of the technique is that the acoustic-elastic variation of wave velocity with stress is extremely small ( $\sim 0.1 \%$ velocity change per GPa of stress).

In this paper, a guided wave ultrasound system has been developed. An indicator model was used to choose the optimal mode which is sensitive to the thermal stress. A large scale rail testbed has been designed to simulate the thermal stress in the real track and establish the relationship between the time delays and the thermal stress. The system was installed on a Chinese high-speed railway from Datong to Xi'an for a test between December 2014 and June 2015. The system operates reliably although some problems involving unreliable 
WCDMA communication have been experienced and realized the on-line monitoring of thermal stress.

\section{Mode selection}

Dispersion curves for guided waves in a CHN60 profile rail are shown in figure 1 . These dispersion curves were computed using the semi-analytical finite element method.
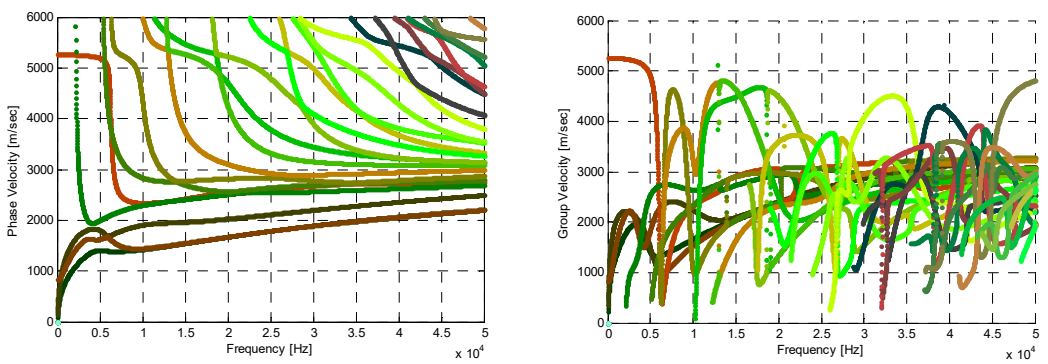

Figure 1: (a) Phase velocity and (b) group velocity dispersion curves of the CHN60 rail.

There are lots of guided wave modes in the range of $0-50 \mathrm{kHz}$. The main problems with using a guided wave are that so many different modes can propagate and all of the modes are dispersive. Therefore, we need to choose one mode that is sensitive to thermal stress.

An indicator model was been used to choose the optimal mode which is sensitive to the thermal stress. The basic factors which determine which mode and frequency to use may be enumerated as follows:

(i) Attenuation;

(ii) Sensitivity;

(iii) Detectability.

We define the indicator model $v$ as follows:

$$
v=\sum_{i=1}^{3} \frac{1}{3} \alpha_{i}
$$

where $\alpha_{i}$ is the each value of the three factors.

\subsection{Attenuation}

Rose et al. [2] used accelerometers as sensors to analysis the attenuation of the guided wave propagating in the rail. The results indicated the relationship between the frequency and the propagating distance (see table 1).

Table 1: $\quad$ Ultrasonic frequency and mode number in the rail.

\begin{tabular}{|c|c|c|c|}
\hline Index & Frequency & Propagating distance & Number of wave modes \\
\hline 1 & $0-20 \mathrm{kHz}$ & $1220 \mathrm{~m}$ & 18 \\
\hline 2 & $20-40 \mathrm{kHz}$ & $1830 \mathrm{~m}$ & 23 \\
\hline 3 & $40-60 \mathrm{kHz}$ & $1964 \mathrm{~m}$ & 33 \\
\hline
\end{tabular}


From table 1, we can figure out that the frequency of a guided wave in the range of $20-40 \mathrm{kHz}$ is a better choice. It has both low attenuation in propagating and few wave modes. Therefore we both consider the propagating distance and the number of modes to define the attenuation factor $\alpha_{1}$. We define $\alpha_{i}$ as follows:

$$
\alpha_{1}=\frac{d_{i}}{d_{3}} \times \frac{n_{1}}{n_{i}}
$$

where $d_{i}$ is the propagating distance of each range of frequency, and $n_{i}$ is the number of wave modes.

Considering the size of the transducer and the installation on the rail, we finally choose $35 \mathrm{kHz}$ as the frequency of the exciting source signal. So the attenuation factor is 0.73 .

\subsection{Sensitivity}

There are about 20 guided wave modes in $35 \mathrm{kHz}$, we define the sensitivity factor of each mode as follows:

$$
\left.\alpha_{2}(i)=\left(\frac{\frac{\Delta v_{i}}{v_{i}}}{\max \left[\frac{\Delta v_{1}}{v_{1}}\right.} \frac{\frac{\Delta v_{2}}{v_{2}}}{\ldots} \frac{\frac{\Delta v_{20}}{v_{20}}}{]}\right]\right)_{\sigma=200 M P a}
$$

We can get the sensitivity factor with the phase velocity of each mode with a thermal stress of $0 \mathrm{MPa}$ and $200 \mathrm{MPa}$. The results are listed in table 2.

Table 2: $\quad$ Phase velocity in different stresses.

\begin{tabular}{|c|c|c|c|c|c|}
\hline Index & $\begin{array}{c}\text { Phase } \\
\text { velocity } \\
(0 \mathrm{MPa})\end{array}$ & $\begin{array}{c}\text { Phase } \\
\text { velocity } \\
(200 \mathrm{MPa})\end{array}$ & $\Delta v_{i}$ & $\frac{\Delta v_{i}}{v_{i}}$ & $\begin{array}{c}\text { Sensitivity } \\
\text { factor }\end{array}$ \\
\hline 1 & 1983.80 & 1988.27 & 4.46 & $0.2251 \%$ & 0.999 \\
\hline 2 & 1984.05 & 1988.52 & 4.47 & $0.2252 \%$ & 1.000 \\
\hline 3 & 2286.06 & 2290.27 & 4.22 & $0.1845 \%$ & 0.819 \\
\hline 4 & 2592.53 & 2597.01 & 4.48 & $0.1729 \%$ & 0.768 \\
\hline 5 & 2725.71 & 2730.27 & 4.56 & $0.1673 \%$ & 0.743 \\
\hline 6 & 2743.72 & 2748.51 & 4.79 & $0.1747 \%$ & 0.776 \\
\hline 7 & 2737.57 & 2741.53 & 3.96 & $0.1447 \%$ & 0.642 \\
\hline 8 & 2919.28 & 2923.39 & 4.11 & $0.1408 \%$ & 0.625 \\
\hline 9 & 3104.71 & 3109.07 & 4.35 & $0.1402 \%$ & 0.623 \\
\hline 10 & 3297.15 & 3301.86 & 4.71 & $0.1428 \%$ & 0.634 \\
\hline 11 & 3389.87 & 3395.58 & 5.71 & $0.1684 \%$ & 0.748 \\
\hline 12 & 3708.61 & 3713.87 & 5.26 & $0.1419 \%$ & 0.630 \\
\hline 13 & 4194.78 & 4201.88 & 7.10 & $0.1691 \%$ & 0.751 \\
\hline 14 & 4167.81 & 4173.74 & 5.93 & $0.1422 \%$ & 0.632 \\
\hline 15 & 4955.74 & 4960.16 & 4.41 & $0.0891 \%$ & 0.396 \\
\hline 16 & 5554.76 & 5557.74 & 2.98 & $0.0537 \%$ & 0.238 \\
\hline 17 & 5862.23 & 5866.93 & 4.69 & $0.0801 \%$ & 0.356 \\
\hline 18 & 6796.88 & 6801.69 & 4.81 & $0.0707 \%$ & 0.314 \\
\hline 19 & 6415.38 & 6420.11 & 4.74 & $0.0738 \%$ & 0.328 \\
\hline 20 & 8155.23 & 8158.97 & 3.74 & $0.0459 \%$ & 0.204 \\
\hline
\end{tabular}




\subsection{Detectability}

Detectability is evaluated by the group velocity measurement error between the experiment data and the curve data. The input signal is five cycle Hanning windowed tone burst with the center frequency $35 \mathrm{kHz}$. Then we get the response signal from transducer about five meters away with the exciting transducer. Finally we compute the group velocity by envelop analysis of each mode.

The result of the selection model is listed in table 4 .

Table 3: Group velocities of response signal.

\begin{tabular}{|c|c|c|c|c|}
\hline Index & $\begin{array}{c}\text { Experiment } \\
\text { data }\end{array}$ & Curve data & $\begin{array}{c}\text { Modes } \\
\text { index }\end{array}$ & $\begin{array}{c}\text { Relative } \\
\text { error }\end{array}$ \\
\hline 1 & 4316.13 & 4266.58 & 16 & $1.16 \%$ \\
\hline 2 & 3019.87 & 3030.30 & 3 & $0.35 \%$ \\
\hline 3 & 2688.55 & 2692.66 & 19 & $0.15 \%$ \\
\hline 4 & 2287.58 & 2230.95 & 11 & $2.54 \%$ \\
\hline 5 & 2098.86 & 2146.2 & 14 & $2.26 \%$ \\
\hline 6 & 1767.68 & 1795.76 & 13 & $1.59 \%$ \\
\hline
\end{tabular}

Table 4: Group velocities of response signal.

\begin{tabular}{|c|c|c|c|c|}
\hline $\begin{array}{c}\text { Modes } \\
\text { index }\end{array}$ & $\alpha_{1}$ & $\alpha_{2}$ & $\alpha_{3}$ & $\tau$ \\
\hline 1 & 0.73 & 1.00 & 0.00 & 0.61 \\
\hline 2 & 0.73 & 1.00 & 0.00 & 0.61 \\
\hline 3 & 0.73 & 0.82 & 0.86 & 0.88 \\
\hline 4 & 0.73 & 0.77 & 0.00 & 0.56 \\
\hline 5 & 0.73 & 0.74 & 0.07 & 0.57 \\
\hline 6 & 0.73 & 0.78 & 0.04 & 0.58 \\
\hline 7 & 0.73 & 0.64 & 1.00 & 0.76 \\
\hline 8 & 0.73 & 0.63 & 0.24 & 0.57 \\
\hline 9 & 0.73 & 0.62 & 0.10 & 0.61 \\
\hline 10 & 0.73 & 0.63 & 0.00 & 0.56 \\
\hline 11 & 0.73 & 0.75 & 0.06 & 0.58 \\
\hline 12 & 0.73 & 0.63 & 0.03 & 0.55 \\
\hline 13 & 0.73 & 0.75 & 0.04 & 0.62 \\
\hline 14 & 0.73 & 0.63 & 0.05 & 0.60 \\
\hline 15 & 0.73 & 0.40 & 0.48 & 0.60 \\
\hline 16 & 0.73 & 0.24 & 0.02 & 0.51 \\
\hline 17 & 0.73 & 0.36 & 0.10 & 0.57 \\
\hline 18 & 0.73 & 0.31 & 0.07 & 0.48 \\
\hline 19 & 0.73 & 0.33 & 0.03 & 0.49 \\
\hline 20 & 0.73 & 0.20 & 1.00 & 0.67 \\
\hline
\end{tabular}

It can be seen from table 4, mode 3 is the optimal mode we need. The wave structure of mode 3 is shown in figure 2. The vibration amplitude in rail head and rail foot is quite small and high in the rail web and the mode we selected propagating in torsional vibration form. 


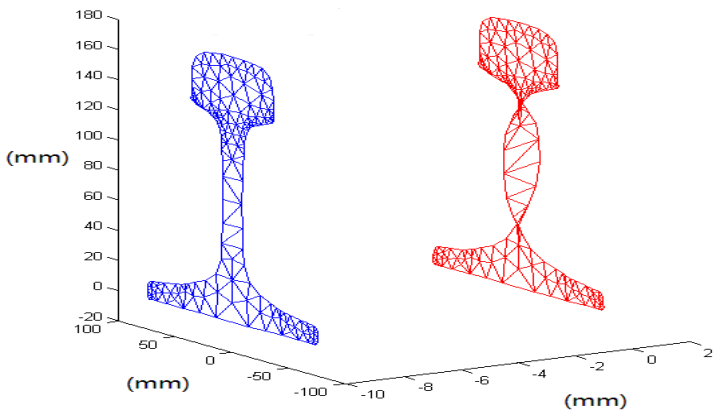

Figure 2: Wave structure of the selected mode.

\section{System calibration}

There are two methods to simulate the stress in the rail, one is to change the temperature of the rail track and compute the stress inside the rail by lock rail temperature. The other one is to apply pressure stress or tensile stress to the free rail, the stress inside the rail can be get from the pressure sensor. It is difficult to control the temperature of rail accuracy, besides the rail temperature inside is quite different from the surface. So the second method was been used to simulate the stress in the rail for system calibration.

A large-scale rail stretch experiment platform has been constructed. The platform (Figure 3) features a 6-meter long full track with CTRS-I rail plate. A hydraulic system was used to actuate the whole platform. It also has two interface load cells to detect the load that the rail applied to.

The platform can apply both the pressure force and tensile force to the rail. We can use the force that actuator apply to rail to simulate the thermal stress when the temperature of the rail does not change.

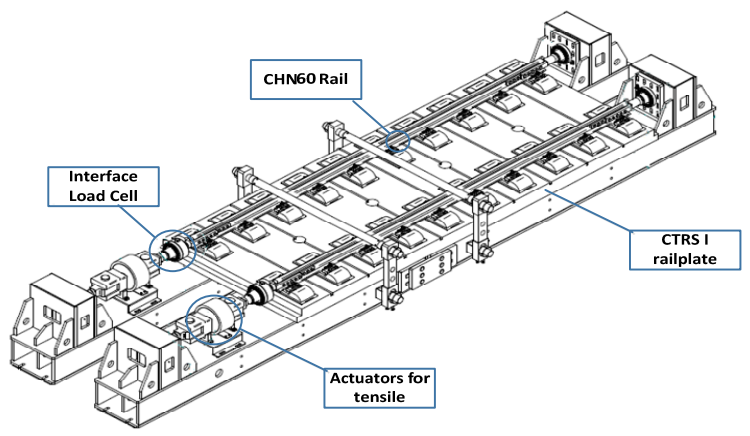

Figure 3: Large-scale rail stretch experiment platform. 
We set a series of stress value and get the time delay between two of transducers, and then we calculate the group velocity of the guided wave to establish the function relationship between the group velocity and the stress. Table 5 shows the results of group velocity and the stress value where the negative value means tensile stress and the positive value means pressure stress.

Table 5: Group velocity and stress value.

\begin{tabular}{|c|c|c|}
\hline Index & $\begin{array}{c}\text { Stress value } \\
(\mathrm{MPa})\end{array}$ & $\begin{array}{c}\text { Group velocity } \\
(\mathrm{m} / \mathrm{s})\end{array}$ \\
\hline 1 & 62.3 & 3018.37 \\
\hline 2 & 54.7 & 3018.31 \\
\hline 3 & 46.9 & 3017.93 \\
\hline 4 & 38.6 & 3018.07 \\
\hline 5 & 29.8 & 3017.87 \\
\hline 6 & 15.7 & 3017.52 \\
\hline 7 & -0.1 & 3017.16 \\
\hline 8 & -18.7 & 3016.99 \\
\hline 9 & -28.3 & 3016.89 \\
\hline 10 & -36.8 & 3016.71 \\
\hline 11 & -45.7 & 3016.43 \\
\hline 12 & -54.9 & 3016.30 \\
\hline 13 & -64.8 & 3016.24 \\
\hline 14 & -73.6 & 3016.16 \\
\hline
\end{tabular}

We use the least square method to fit the data and get the linear fitting curve which indicates the function relationship between stress and group velocity.

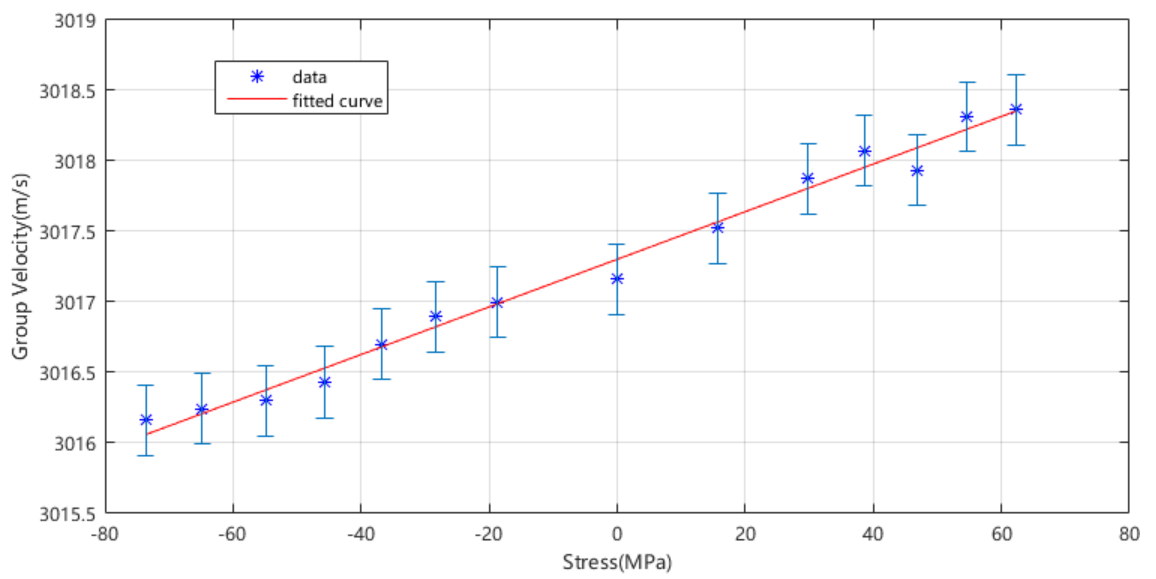

Figure 4: Fitting curve with error bar of stress vs group velocity. 


\section{Installation and results}

The online monitoring of thermal stress system concept is illustrated in figure 5 . The system makes use of permanently installed piezoelectric transducers to transmit and receive guided waves in rail.

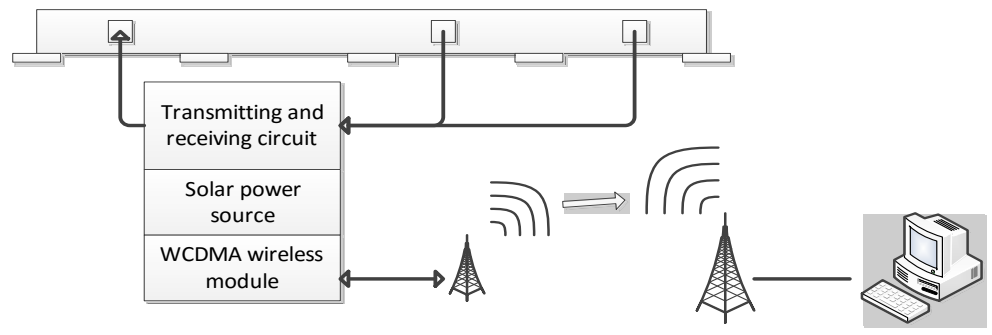

Figure 5: Concept of online monitoring of a thermal stress system.

The piezoelectric transducers are dry-coupled to the rail by epoxy glue and held by a metal holder which is fixed on the rail foot by bolt. The circuit which operates continuously is powered by solar panels. Transducers and electronics are EMI isolated to ensure the accuracy of the data acquiring and transmitting. The data received transmit to a server via WCMDA network. The information is recorded and saved in the remote computer terminal.

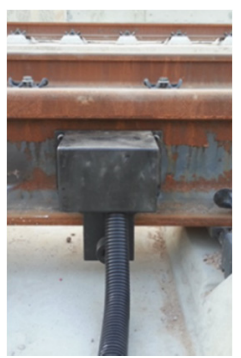

(a)

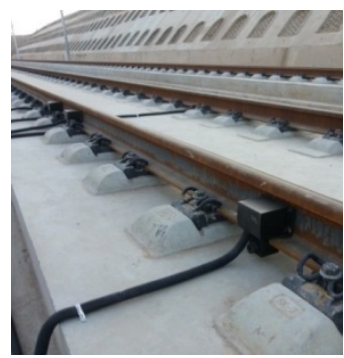

(b)

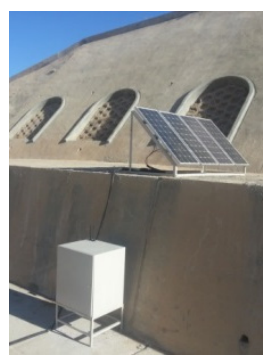

(c)

Figure 6: (a) Piezoelectric transducer and holder; (b) layout of transmit and receive transducer; (c) solar panel and receive station.

The installation of the system was completed at Datong-Xi'an high-speed railway in December 2014. We totally installed three monitoring stations along a $5 \mathrm{~km}$ rail. It took one week to complete the installation and adjust the whole system's communication with the remote computer terminal. The system was programmed to monitor the rail every 5 minutes. There were also some problems with the WCDMA network because the place of installation was in a valley. There were few equipment failure occurrences. The system performed quite well 
in the period from December 2014 to June 2015. Figure 7 illustrates the performance of the system.

The data transmitted from the receive station is shown in figure 8. A Butterworth filter was designed to filter the data received. Then we applied envelop analysis to the data to compute the group velocity of the guided wave in the rail. Figure 9 shows the signal received from two transducers.

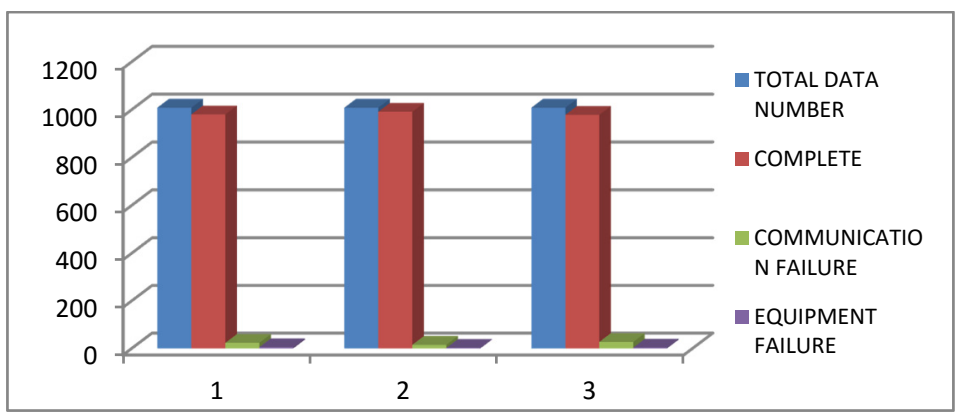

Figure 7: Performance of the system.
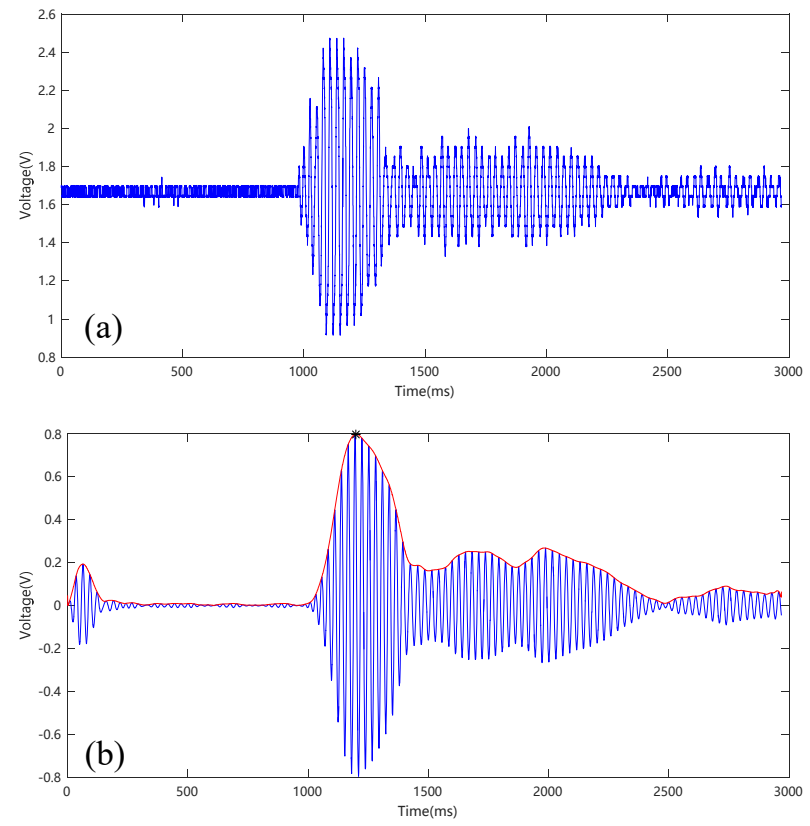

Figure 8: (a) Unfiltered signal; (b) filtered and enveloped signal. 

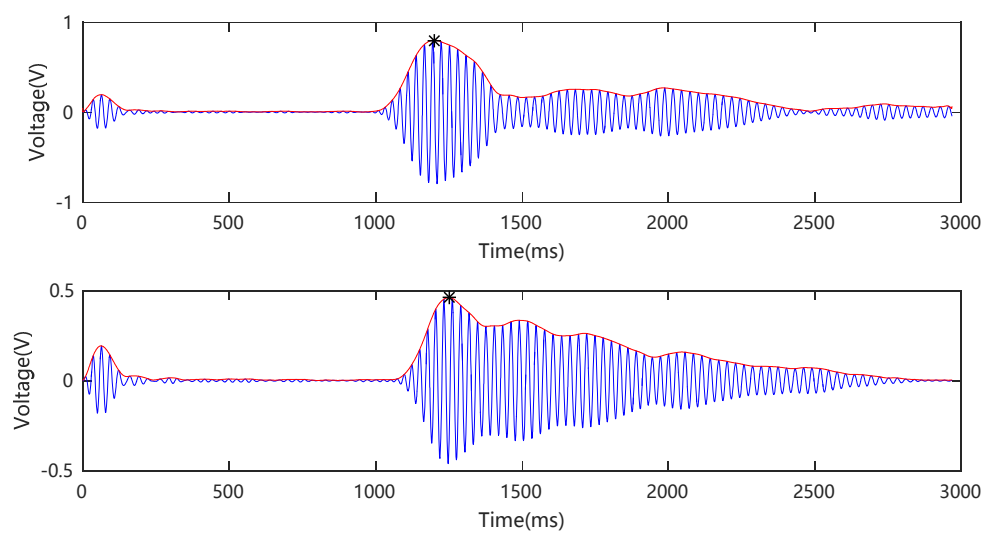

Figure 9: Signal from two transducers.

After analysis of the data transmitted from the receive station, we can find the curve of the thermal stress inside the rail (see figure 10). We can figure out that thermal stress the system got is well fitted with the rail temperature. Because of unknown of the lock temperature of the railway, the accuracy of the detection cannot be evaluated.
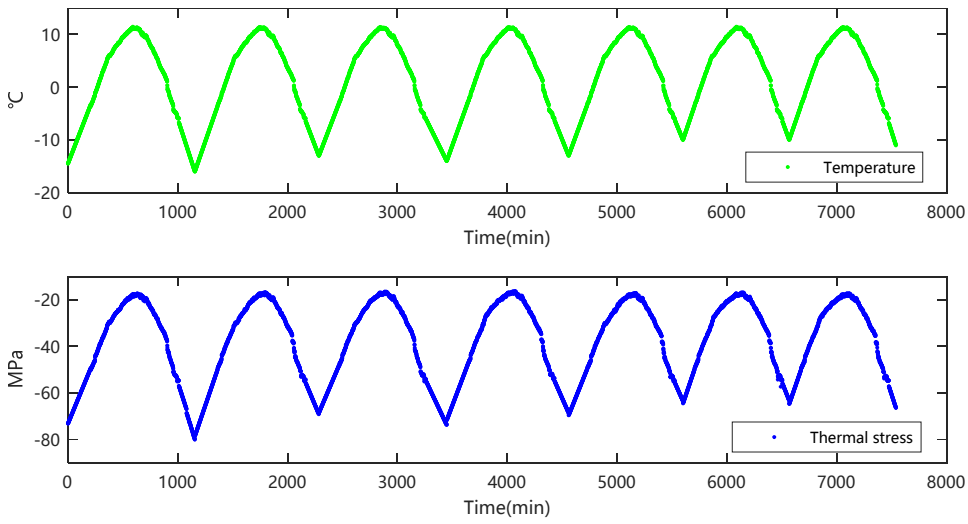

Figure 10: Temperature and thermal stress curve.

\section{Conclusion}

The system has been installed for six months and good results have been found. The results indicated that the system is feasible and reliable even though we found some problems with the communication. Some more improvement which focuses on the accuracy and distance cover will be done in the future. 


\section{References}

[1] J. Li, J.L. Rose, Implementing guided wave mode control by use of a phased transducer array, Ultrasonics, Ferroelectrics, and Frequency Control, IEEE Transactions on, 48, pp. 761-768, 2001.

[2] J.L. Rose, M.J. Avioli, P. Mudge, R. Sanderson, Guided wave inspection potential of defects in rail, NDT \& E International, 37, pp. 153-161, 2004.

[3] I. Bartoli, A. Marzani, F.L. di Scalea, E. Viola, Modeling wave propagation in damped waveguides of arbitrary cross-section, Journal of Sound and Vibration, 295, pp. 685-707, 2006.

[4] F. Chen, P.D. Wilcox, The effect of load on guided wave propagation, Ultrasonics, 47, pp. 111-122, 2007.

[5] P.W. Loveday, Semi-analytical finite element analysis of elastic waveguides subjected to axial loads, Ultrasonics, 49, pp. 298-300, 2009.

[6] I. Bartoli, S. Coccia, R. Phillips, A. Srivastava, F.L. di Scalea, S. Salamone, M. Fateh, G. Carr, Stress dependence of guided waves in rails, Health Monitoring of Structural and Biological Systems, Proc. SPIE 7650, International Society for Optics and Photonics, San Diego, California, USA, pp. 765021-765010, 2010.

[7] F.A. Burger, A practical continuous operating rail break detection system using guided waves, 18th WCNDT, 2012.

[8] Xining $\mathrm{Xu}$, Baoqing Guo, Zujun Yu, Hongmei Shi, Liqaing Zhu, Semi-analytical finite elements method for calculating dispersion curves of ultrasonic guided waves in a rail, Chinese Journal of Scientific Instrument, 35, pp. 2392-2398, 2014. 